Juan José Castillo, jicastillo@cps.ucm.es y Pablo López Calle plopezca@ucm.es

Resumen

\title{
LA JUVENTUD ENTRE CATEGORÍA SOCIAL Y COARTADA IDEOLÓGICA
}

En las investigaciones que hemos realizado en las últimas décadas en España nos hemos centrado, por una parte, en la organización del período juvenil como mecanismo de transformación de los modelos productivos. Por otra parte hemos estudiado la utilización de la juventud como grupo social (que no lo es) como coartada ideológica para realizar las grandes reformas laborales de los años ochenta y noventa, e intensificar así la reproducción de las desigualdades sociales. Estos procesos quedan ocultos bajo el fetichismo de la juventud como proceso de metamorfosis formal en el que el estatus asdscrito se convierte misteriosamente en estatus adquirido.

Palabras Clave: juventud, reproducción social, modelos productivos, crisis y precariedad.

\section{Abstract \\ YOUTH : A SOCIAL CATEGORY AND AN IDEOLOGICAL ALIBI}

Over the last twenty years, we have been focusing our investigation on two main topics: on the one hand, the analysis of the transformation of the productive models through the integration of new generations within these models. On the other hand, we have studied the consideration of youth as a social group (which is not) as an ideological alibi to carry out the great labor reforms of the 1980s and 1990s, thus intensifying the reproduction of social inequalities. These processes are hidden under the fetishism of youth as a process of formal metamorphosis in which the inherited status mysteriously becomes acquired status.

Key words: Youth, social reproduction, productive models, crisis and precariousness.

\section{LA JUVENTUD ENTRE CATEGORÍA SOCIAL Y COARTADA IDEOLÓGICA}

\section{Introducción.}

“Esta es nuestra hipótesis de partida: la 'juventud' no forma un grupo social. Bajo la identidad del nombre 'juventud'-bajo la presunta identidad de todos los incluidos en un arco de edades- se agrupan sujetos y situación que sólo tienen en común la edad"

(Martín Criado, 1998:15).

A pesar de la cita de inicio, la juventud, entendida como una institución social, es decir, como proceso estructurado de incorporación al mundo productivo de las nuevas generaciones y como organización del proceso de reproducción del sistema social, se revela como un objeto de estudio sociológico particularmente pertinente y relevante 
para analizar la evolución y transformación de los modelos productivos en el medio y largo plazo.

No obstante, es cierto que la juventud, en sí misma, no es un grupo social. Lo joven, ni remite a un lugar en la estructura social; ni a una posición respecto de la propiedad de los medios de producción; ni siquiera la juventud está sometida de forma homogénea a formas de discriminación o exclusión social que la identifiquen frente a otros grupos sociales. Los jóvenes como grupo sólo comparten generación, en lo demás son altamente heterogéneos.

De hecho, en muchas ocasiones precisamente la consideración de la juventud como un grupo social (un grupo con unos rasgos y una posición determinados en la estructura social) más bien ha tenido un efecto naturalizador de procesos de reproducción de la estructura de clases dentro de cada generación. Por ejemplo, la alta temporalidad y los bajos salarios de los jóvenes se asocian en muchas ocasiones a su falta de experiencia y antigüedad. De manera que, por una parte, la precariedad de los jóvenes, en cierta forma, excluye hablar de la precariedad del trabajo (la precariedad no es la condición del empleo sino un estado por el que pasan los trabajadores noveles, y en ese sentido es una parte de la estabilidad). Por otra parte hay diferencias bastante más importantes en cuanto a condiciones de vida y trabajo entre jóvenes con diferente estatus social que entre jóvenes y mayores.

En este texto vamos a tratar de explotar estas dos dimensiones de lo que podemos llamar el carácter fetichista de la juventud como institución, su capacidad para desvelar y ocultar determinados procesos sociales vinculados a la reproducción y transformación del sistema social.

Por ejemplo, la organización social de la transición a la vida adulta configura las condiciones de incorporación al mercado de trabajo de las nuevas generaciones y asimila con ello las contradicciones y los procesos de alienación que atraviesan las relaciones salariales. En la medida en que el fetichismo del trabajo-mercancía consiste en presentar las determinaciones estructurales de salario y empleo como si fueran preferencias de cada trabajador, la juventud, como período de tránsito de la niñez a la vida adulta, es el período en el cuál se hacen efectivos los mecanismos de la reproducción de las desigualdades de clase, pero en el que se presentan esas determinaciones marcadas por el origen social como si fueran preferencias electivas de cada individuo.

La juventud es la línea imaginaria que separaría la etapa de la vida en la que el estatus social del sujeto corresponde al de la familia de origen, de la etapa en la que éste se origina en su esfuerzo individual. El período juvenil sería algo así como el proceso de metamorfosis del "hombre de destino" en el "hombre de carácter" benjamianos. La etapa de juventud está organizada por sistemas de igualación de oportunidades, tales como el sistema de educación público, así como por la provisión de beneficios sociales y becas de estudios a los económicamente desfavorecidos que demuestren un mayor esfuerzo por "labrarse su futuro". No obstante, como es sabido, esos sistemas de formación son también sistemas de selección meritocrática mediante la evaluación estándar de conocimientos. Sistemas que no hacen sino certificar, pasar a limpio o blanquear, los diferentes recursos para alcanzar méritos académicos que provee el llamado "currículum oculto" [Bourdieu y Passeron, 1970].

Las instituciones que promueven la igualdad de oportunidades, fundamentalmente la educación pública, aseguran -al menos formalmente- que es en este período donde el joven tiene la opción de aprovechar o desaprovechar las oportunidades de movilidad de 
clase, tanto para ascender como para descender en la jerarquía social (Martín Criado, 2010). Pues además de ser un período esencialmente formativo -en el que se acumulan las credenciales que otorgarán un lugar de salida en la llamada "cola" del mercado de trabajo", al mismo tiempo es una etapa característicamente plena de libertades y oportunidades en la esfera del ocio y el consumo. De hecho las dinámicas de diferenciación social fraguadas en esta etapa producen ya un tipo de desigualdad Rawlsiana que no sólo es formalmente justa y equitativa, sino que es necesaria precisamente para dar sentido y contenido a la libertad y la igualdad individual: al igual derecho de acceso a la propiedad por el trabajo. La libertad juvenil proporciona un grado mayor de responsabilidad en el éxito o el fracaso social, a medida que el espacio de oportunidades electivas en que se forja se amplía.

Es una etapa, además, en la que los recursos culturales para la integración social diferencial al mundo del trabajo y la conformación de expectativas y estrategias profesionales no vienen exclusiva o directamente provistos por la familia, aunque operan como factores igualmente fuertes de reproducción de clase, sino que están mediados por las culturas de clase transmitidas y compartidas por los pares, a través de mecanismos de reconocimiento y aceptación. Culturas de clase que, a pesar de tener una alta carga adscriptiva, tienden a presentarse con un alto componente preferencial, más si cabe cuando se presentan como "contraculturas obreras" [Willis, 1988]-, contraculturas en las que el grado de penetración cultural, de resistencia a las culturas meritocráticas y del emprendizaje de la clase media, y de apropiación de esos contravalores como propios, actúan precisamente no sólo como mecanismos de reproducción de clase, sino que son una limitación para la movilización colectiva en aras de la transformación del sistema. Es como si también a través de la adscripción a estas culturas los jóvenes se hicieran cargo de su destino, como si éste fuera ya producto de su propio carácter.

En definitiva, además de ser un período vital clave para analizar los mecanismos de reproducción de clase en términos generacionales, cuando de lo que se trata es de estudiar transformaciones sociales a medio y largo plazo del sistema social, la población joven se revela como un analizador privilegiado, pues es evidente que las condiciones de vida y de trabajo en las que se incorpora cada nueva generación al ámbito productivo determinan las posibilidades de desarrollo de dicho sistema en el futuro.

En las investigaciones que hemos realizado en las últimas décadas en España [Castillo y López Calle, 2004, 2007] nos hemos centrado en estas dos dimensiones de la juventud en España: por una parte analizando cómo la transformación del modelo productivo se ha operado mediante la incorporación de las nuevas generaciones con otras condiciones de trabajo que las de sus padres, a partir de las grandes reformas laborales de los años ochenta y noventa, y por otra, cómo, al tiempo que se transforma el modelo de desarrollo, se reproducen las relaciones de clase y se ocultan dichos mecanismos de reproducción.

\section{2. "Los hijos de las reformas laborales"}

El origen del modelo productivo español actual hay que buscarlo en las reformas laborales y los procesos de reorganización productiva que se ponen en marcha a partir de los años ochenta. De dichas reformas, las dos principales se produjeron en el año 1984 y 1994. Éstas se justificaron por la necesidad de resolver la crisis de desempleo provocada por las reconversiones industriales de las décadas precedentes y la consiguiente desinversión de capital en sectores tradicionales de la industria pesada 
(minería, siderurgia, bienes de equipo, etc.). Estas reformas se orientaron, fundamentalmente, y siguiendo las directrices de los planes de competitividad europeos, a la flexibilización de las formas de contratación como principal medicina contra el paro ${ }^{1}$.

Se puede caracterizar a la primera reforma como la de la flexibilización externa, o del empleo, (abaratamiento del despido, nuevas formas de contratación y contratos especiales para la inserción de grupos desfavorecidos), y a la segunda, como la reforma de la flexibilización interna, o del trabajo, (de incidencia clave en la organización del trabajo y en los convenios colectivos).

\section{La juventud como coartada ideológica de las reformas}

El paro juvenil fue utilizado como coartada ideológica para la precarización del mercado de trabajo, puesto que fue a través de la renovación de la fuerza de trabajo como se produjo la transformación del mismo: el obrero tradicional no perdió 'directamente' sus derechos adquiridos, o fue indemnizado por su pérdida, pero sí lo hizo el obrero colectivo a través de formas excepcionales de contratación de los nuevos trabajadores que ponían en cuarentena esos derechos. La mayoría de las nuevas formas de contratación estaban destinadas a facilitar la incorporación al mercado de trabajo de los jóvenes desempleados, o más bien de los jóvenes inempleados que accedían a un puesto de trabajo por primera vez, sobre el supuesto de que el problema del empleo era la excesiva rigidez de un modelo de relaciones laborales que era incoherente con las nuevas formas de producción, basadas en la fabricación flexible y la rápida adaptación a los cambios en la demanda de bienes y servicios.

No obstante, ya a finales de los ochenta, en pleno período de reformas, algunos autores activaron la alarma sobre la posibilidad de que el desempleo juvenil se estuviera utilizando más bien como coartada ideológica legitimadora para facilitar la transformación hacia ese tipo de modelo productivo basado en la intensificación del trabajo que iba a requerir la individualización de las relaciones laborales como forma de articulación de las relaciones salariales ${ }^{2}$.

\footnotetext{
${ }^{1}$ En palabras de José Ignacio Pérez Infante, Director General de Empleo en 1989, y Lorenzo Cachón Rodríguez, Subdirector General de estudios de Empleo y Mercado de Trabajo, " La política de empleo puesta en marcha con la reforma del Estatuto de los trabajadores en 1984 pretendía eliminar incertidumbres dentro del campo institucional y normativo del mercado laboral, creando un cuadro de figuras contractuales de mayor claridad, estabilidad y flexibilidad [...] La política de empleo juvenil se articula en la actualidad en torno tres ejes que pretenden: 1. Mejorar la formación profesional de los jóvenes, y a ello van dirigidas las actividades del Plan de Formación e Inserción Profesional (FIP); 2. Posibilitar su inserción en el mercado laboral, mediante contratos en formación o en prácticas, que proporcionen las primeras experiencias laborales a los jóvenes, y 3. Facilitar la integración laboral mediante la incentivación de contratos de duración indefinida". Pérez Infante y Cachón Rodríguez: "La política de empleo juvenil en la España actual”, en Torregrosa et al., 1989: 206-224, p. 207.

2 "De este modo, los jóvenes, junto con otros colectivos (minusválidos, parados que no perciben subsidios de desempleo, etc.). Van a constituirse en los destinatarios de una serie de medidas de carácter excepcional. A la vez van a ser la punta de lanza de la flexibilización del mercado de trabajo. Ellos van a ser la coartada sobre la que se argumentará la quiebra de la relativa estabilidad y seguridad del mercado de trabajo, rebautizadas, ahora, con las rigideces que impiden la integración de los nuevos elementos. Como toda coartada ideológica, encierra un silogismo irrefutable: de no alterarse la lógica del proceso de acumulación, resulta evidente que sólo desestabilizando a los que están, cabe integrar a los que no están. Y en la medida en que esta lógica aparece desprovista de fundamentación histórico-social, esta coartada argumental se materializa en la creciente división entre los que están y los que no están". [Bilbao, 1989:63].
} 
Sería esta consideración, sospechaban, la que habría de provocar precisamente el incremento de la vulnerabilidad de la clase trabajadora en general y de los jóvenes en particular $^{3}$. Casi se podría decir que los problemas actuales específicos de los trabajadores adultos que hoy configuran el nuevo precariado comienzan con una reforma hecha para "ayudarles" .

La respuesta a estas iniciativas, defendidas de forma extensa e intensa en esos años terminó, provisionalmente, con una huelga general, convocada por los principales sindicatos españoles, uno de cuyos ejes centrales, además de otras medidas de carácter muy general, fue, precisamente, la oposición frontal a estos contratos y medidas excepcionales y dañinas para los jóvenes, como se decía, con una visión muy acertada del carácter de precarización general de las relaciones laborales para todos y todas las trabajadoras.

Junto a las nuevas formas de contratación orientadas a la flexibilización de las relaciones laborales también se había empezado a promocionar, ya entonces, la nueva cultura del emprendimiento y la activación personal basadas en el esfuerzo y el mérito individual como única y principal forma de reparto de la propiedad social. Nosotros mismos firmábamos estas líneas en la introducción a un número monográfico de la Revista Española de Investigaciones Sociológicas dedicado "Crisis del trabajo y cambios sociales", donde los jóvenes eran un analizador preferente, en 1987: "En la Comunidad Económica Europea, el desempleo juvenil se plantea como «prioridad absoluta» y ha dado lugar a la puesta en marcha de una serie de resoluciones y medidas, con un alto grado de aceptación por parte de los actores sociales, que si hubieran de resumirse en una frase corta podría recogerse ésta sobre cómo deben ser los jóvenes ante el trabajo, en la situación actual: deben «tener un espíritu despierto, estar informados y dispuestos a adaptarse a lo nuevo, ser creativos y emprendedores»; deben aprovechar las reorganizaciones del tiempo de trabajo; estar abiertos a nuevas formas de trabajo y actividad; darse como objetivo un trabajo de colaboración social, de restauración del patrimonio, etc.; circular entre el sistema productivo y el educativo; no

\footnotetext{
3 "Las políticas de empleo sobre los jóvenes diseñan cambios en perspectiva de futuro, creando los marcos que van a moldear, con cierta capacidad de influencia las posibilidades de desarrollo de las imágenes del trabajo". [Castillo, 1989:75]

-"En cada momento histórico -y por supuesto, con mayor intensidad en los momentos de cambio- la juventud experimenta las transformaciones que van produciéndose en la sociedad; puede decirse que la juventud es el laboratorio y el escenario principal del cambio de las estructuras sociales" [Zárraga, 1984:13]

${ }^{4}$ El contrato en prácticas permitía pagar un salario hasta un $60 \%$ menor para el mismo puesto durante dos años. Se crearon diferentes fórmulas de trabajo mediante becas de formación y prácticas empresariales, de las que se abusa con enorme frecuencia en España (Reconocido por la propia comunidad Europea Council recommendation on a Quality Framework for Traineeships, 2014.

http://www.consilium.europa.eu/uedocs/cms_data/docs/pressdata/en/lsa/141424.pdf. Formas de trabajo para jóvenes que no se consideran empleos plenamente, provistos de todos los derechos laborales, sino tareas a caballo entre las prácticas y la formación (excluyendo al trabajador, en muchos casos, del derecho al desempleo y a la seguridad social y percibiendo parte del salario en concepto de adquisición de experiencia o pericias). Los contratos para la formación, en principio sólo para menores de 21 años, pero con muchas excepciones, no estipulaban condiciones salariales desventajosas por hora trabajada, pero no podían durar más de dos años, con lo que muchas empresas sólo los han utilizado para acceder a esas ventajas fiscales y rescindir la relación laboral terminado el plazo. Los contratos de inserción, para gente desempleada, sólo realizable por entidades públicas y sin ánimo de lucro, no estaban siquiera limitados por el salario mínimo.
} 
tener una mentalidad «atrasada» de asalariados; no buscar un trabajo para toda la vida; no pedir salarios (o retribuciones) «más allá de lo posible»; crear su propia empresa..."5.

Sin embargo, advertíamos ya entonces nosotros mismos: "Las políticas de empleo juvenil pudieran ser un lugar donde la coincidencia en la aprobación de las actuaciones públicas nos podría indicar cuál será el camino más importante elegido para los trabajos, empleos o las actividades de mañana. Por supuesto, no sólo para los jóvenes, sino probablemente como horizonte -en un plazo medio- que cubra a la mayor parte de los trabajadores".

[Porque, continúa la auto-cita,] "el argumento que sostiene que más vale un empleo sin algo [para los jóvenes] de lo que antes le era consustancial, que nada, es uno de los puntos nodales de la versión «flexibilizadora» de estas transformaciones: más vale el dualismo construido y controlado que el ser víctimas incontroladas de la economía sumergida. Así, por ejemplo, se han alzado voces en nuestro país pidiendo que «ordenadamente» se establezca un salario mínimo más mínimo para los jóvenes hasta los veintiocho años"

Y, poco antes, habíamos adelantado, en una tribuna de la sección de Economía de $E l$ País, en abril de 1986, que las políticas para los jóvenes eran, en realidad, políticas de destrucción de la eventual actuación conjunta de colectivos de trabajadores: unos fuera del mercado de trabajo por viejos, y los otros por jóvenes, "Ante la urgencia de abrir el mercado del trabajo a las nuevas generaciones, la tercera edad comienza ahora a poco más de los 35 años, mientras que hasta los 30 todo trabajador es un contratable en precario. Lo que hoy necesitan los empresarios son trabajadores débiles en el mercado del trabajo y fuertes en los procesos de producción".

Antes bien, en aquellas reformas, los problemas propios de la juventud fueron imputados a un estadio pasajero, propio de la edad, del contexto histórico y los avatares demográficos (léase el baby boom de los setenta), que ocultaban así problemas estructurales de la evolución del mercado de trabajo y de la organización productiva en nuestro país. Con ello se ocultaban también las fuertes diferencias y desigualdades internas de clase bajo un enfrentamiento entre grupos de edad adscritos a mercados primarios y secundarios. [Zárraga,1985] outsiders contra insiders. De forma que la división de clases entre las generaciones fragmenta los movimientos juveniles, pero la fragmentación entre generaciones frena la movilización de las clases.

\section{El llamado "milagro económico español": un modelo de crecimiento basado en "la esquilmación de una generación"}

En el año 2003, realizábamos una investigación sobre Los hijos de las reformas laborales [Castillo y López Calle, 2004] encargada por el sindicato UGT basada en una treintena de entrevistas en profundidad a jóvenes de diferentes edades y perfiles sociolaborales que nos llevaba a concluir, en líneas generales, que el modelo productivo de los años del "milagro económico español", entre finales de los noventa y principios del nuevo milenio, se basaba en un modelo de vía baja de desarrollo basado en la explotación de recursos técnicos y humanos por encima de las posibilidades de reproducción del sistema. Y caracterizábamos esta suerte de sobreexplotación de

\footnotetext{
${ }^{5}$ Las citas corresponden al informe de la Comunidad Europea sobre Juventud y empleo de septiembreoctubre 1986, p. 26. y el artículo a Castillo, Juan José: “Introducción”, REIS, 38, 1987 p. 16.

${ }^{6}$ Ibidem, pp. 15-16 de la REIS 38,1987.
} 
recursos humanos como "la esquilmación de una generación" [Castillo y López Calle, 2007]

En primer lugar las causas de este proceso de esquilmación que estábamos detectando eran las siguientes: se había producido una fragmentación del obrero colectivo por edades a partir de estrategias empresariales de externalización y subcontratación hacia el sector servicios y la emergencia de empresas de servicios especializadas en la contratación de jóvenes en precario ${ }^{7}$. Ello había dado lugar a la balcanización de los mercados de trabajo y sectores por edades, lo cual había supuesto un cambio de la movilidad vertical por la movilidad horizontal de los trabajadores (imposibilidad de construir carreras profesionales y de acceder a puestos de mayor cualificación y tareas de mayor valor añadido). También ello estaba vinculado al incremento de la sobrecualificación de los trabajadores jóvenes (jóvenes cada vez más formados para puestos cada vez más descualificados) $)^{8}$. Por último, ello había dado lugar también a un proceso de intensa atomización empresarial ${ }^{9}$ y por supuesto, a un proceso de individualización de las relaciones laborales de los trabajadores jóvenes, a lo que se añadía, por las características del empleo y de las empresas en las que trabajaban, la imposibilidad creciente para tener representación sindical en las empresas ${ }^{10}$.

En segundo lugar, los síntomas de esta suerte de modelo de crecimiento basado en la sobreexplotación de los recursos humanos por encima de las posibilidades de reproducción del sistema se evidenciaban ya entonces a partir del análisis de diversos indicadores. En primer término, la orientación de los sectores especializados en la contratación de jóvenes hacia estrategias de rentabilidad basadas en los bajos costes y la intensificación del trabajo se manifestaba en el incremento de los índices de siniestralidad laboral, que superaban en el año 2003 entre los trabajadores jóvenes tasas del 96 por mil (frente a una tasa media ya de por sí alta respecto a la media europea del 55 por mil). Además, los accidentes de trabajo se concentraban en actividades ocupadas por jóvenes, en empresas de menos de 50 trabajadores, sobre trabajadores que llevaban menos de un año en sus empresas y cuadruplicaban su incidencia en los contratados temporalmente. En definitiva, por cada trabajador mayor de 50 años, cinco jóvenes se accidentaban $^{11}$. A lo cual había que añadir la preocupante evolución de otro tipo de

\footnotetext{
${ }^{7}$ Según la Encuesta de Población Activa el empleo de los jóvenes se precarizó rápidamente: ya en el año 2002 , el $80 \%$ de los trabajadores menores de 30 años y el $92 \%$ de los menos de 25 años tenía un contrato de tipo temporal. Una cifra extremadamente alta si se comparaba con la media europea de la época (38\%). El 50\% de estos trabajadores jóvenes había conocido ya al menos tres empleos remunerados en el momento de la encuesta

${ }^{8} \mathrm{Si}$ en 1994, el porcentaje medio de los trabajadores que declaraban sentirse sobrecualificados se situaba en el 47\%, este índice había subido nada menos que veinte puntos en una década ( 7 de cada 10 jóvenes reconocen que su trabajo no está relacionado con su formación) [INJUVE, 2001]. Según la Encuesta de Población Activa, entre 1987 y 2008, si bien se observa un descenso porcentual muy significativo entre los ocupados con un bajo o nulo nivel de cualificación, pasando del $58 \%$ al $15 \%$, crecen, sin embargo, las ocupaciones no cualificadas, pasando del 5,2\% en 1987 al 18\% en 2007.

${ }^{9}$ El tamaño medio de las empresas en 2007 era de sólo 11 trabajadores. Las empresas de menos de 10 asalariados representaban el $94 \%$ de las empresas españolas y reagrupaban al 50,5\% de los asalariados. La esperanza de vida media de las empresas también era muy reducida: según el Directorio General de Empresas, elaborado por el INE, el $15 \%$ de las empresas españolas tenía menos de un año de antigüedad. Era una época en la que cada año 300.000 nuevas empresas abrían sus puertas y 250.000 las cerraban, sobre un total de 2,6 millones para todo el país.

${ }^{10}$ En el año 2009 más de un $80 \%$ de los jóvenes trabajadores menores de 35 años nunca había pertenecido a un sindicato. (CIS, 2009);

${ }^{11}$ INSTITUTO REGIONAL DE SEGURIDAD Y SALUD EN EL TRABAJO DE LA COMUNIDAD
} 
indicadores que, considerados como una especie de punta del iceberg de la degradación de las condiciones de empleo y trabajo en las relaciones laborales cotidianas, también se estaban incrementando. Por ejemplo, las enfermedades y dolencias entre los jóvenes de tipo mental. Pues la creciente vulnerabilidad de los trabajadores en sus puestos, la citada individualización de las relaciones laborales, unidos a la intensificación de los sistemas meritocráticos de estratificación social, estaban derivaban en una especie de interiorización de esos problemas en clave de culpabilización personal que tenían como correlato, en los casos más extremos, la aparición de episodios psicopatológicos tales como la ansiedad, el estrés o la depresión ${ }^{12}$.

Pero también, junto a esos efectos sobre el estado físico y mental de los propios trabajadores, evidenciábamos otro conjunto de síntomas relacionados con las limitaciones a la autonomía, la integración y la participación social de los jóvenes: la temporalidad en el empleo y los bajos salarios estaban provocando, en primer lugar, el retraso de la edad media de emancipación ${ }^{13}$, el retraso la edad media al formar una familia y de la edad al tener el primer hijo. Y por tanto, estaban provocando también la caída de las tasas de fecundidad ${ }^{14}$. De manera que, en esos años, un joven español que quisiera emanciparse y vivir solo, debería dedicar el $88 \%$ de sus ingresos a pagar su vivienda. A finales de la primera década del siglo el 53,9\% de los jóvenes empleados entre 25 y 29 años no vivían exclusivamente de sus ingresos, porcentaje que alcanzaba al 80,8\% en los que tenían entre 20 y 24 años, y que el 14,6\% de los jóvenes de entre 25 y 29 años se mantenían de los ingresos de otras personas, situación que afectaba al 38,5\% de los de 20 a 24 años. [Consejo de la Juventud de España, 2008].

De este modo, y cerrando el conjunto de efectos que analizábamos sobre el citado modelo de crecimiento basado en el uso de recursos futuros, la financiarización de la economía que sufrió nuestro país en esos años está conectado con el fuerte endeudamiento familiar derivado de la diferencia entre las necesidades de reproducción de la fuerza de trabajo y las condiciones de empleo y trabajo, especialmente de los jóvenes ${ }^{15}$. El incremento de la demanda privada de financiación, que estuvo básicamente destinada a financiar el acceso a la vivienda de las nuevas generaciones,

DE MADRID, Plan Director en prevención de riesgos laborales de la Comunidad de Madrid 2002-2003. Madrid, UGT-Madrid, 2002.

${ }^{12}$ Un $8 \%$ de los jóvenes españoles sufrían depresión; afectando más a mujeres que a hombres; y más a los jóvenes de familias con menor nivel de renta. De forma que el suicidio por depresión era la primera causa de muerte en la población adolescente. A este problema específico dedicamos posteriormente un artículo basado en uno de los casos que habíamos entrevistado [López Calle, 2012].

${ }^{13}$ Cuatro de cada cinco jóvenes menores de 25 años seguían viviendo en casa de los padres a principios del año 2000. Situación que se agravaba en el año 2009, cuando uno de cada cuatro trabajadores entre 30 y 34 años no había logrado la emancipación económica, doblándose esta proporción en el caso de los comprendidos entre los 25 y los 29 años, según datos del Obser6vatorio de Vivienda en España del Consejo de la Juventud de 2010 las características definitorias de la población menor de 35 años eran el bajo porcentaje de hogares jóvenes (12’0\%) y la baja tasa de emancipación (31'7\%).

${ }^{14}$ Por ejemplo, entre 1975 y 2000, en la Comunidad de Madrid la tasa de fecundidad cayó del 2,8 al 1,2 (uno de los índices sintéticos de fecundidad más bajos del mundo). La edad media de la madre al tener el primer hijo subió de los 25 a los 32 años. La edad media al matrimonio pasó de los 26 años a los 32 . Y el crecimiento natural de la población se redujo de los 63.921 de 1975 a los 11.148 del año 2000. INSTITUTO DE ESTADÍSTICA DE LA COMUNIDAD DE MADRID, Boletín de Demografía y Salud, $\mathrm{n}^{\circ} 65,(2004)$.

${ }^{15}$ Según la Encuesta Financiera de las Familias del Banco de España la deuda familiar en sólo seis años pasó del ya de por sí alto índice de endeudamiento del 79\% de la renta disponible en 2002, a nada menos que $128 \%$ en 2008 
estaba alimentando, a su vez, el incremento de los precios de las viviendas, y el incremento de la demanda de préstamos: la denominada burbuja inmobiliaria [López Calle e Ibáñez Rojo, 2012]. De manera que, a su vez, el incremento constante de los precios de los pisos alimentaba un modelo de crecimiento basado en actividades de escaso valor añadido dedicados a contratar trabajadores jóvenes y descualificados, tales como la construcción.

Paradójicamente, el fuerte endeudamiento familiar será precisamente una de las principales coartadas o acusaciones que se volcarán sobre la población por parte del discurso oficial con el advenimiento de crisis económica. Pues existe ya una especie de consenso en la opinión pública -o publicada- sobre la idea de que el devenir de dicha crisis se debe, en gran parte, a que la ciudadanía ha "vivido por encima de sus posibilidades". Siendo este diagnóstico, por otra parte, toda una declaración de intenciones acerca de las medidas de ajuste que habrá que tomar para salir de la misma.

\section{Concluyendo: la crisis como resultado de un modelo de crecimiento insostenible. Los jóvenes (algunos jóvenes) como principales damnificados}

Curiosamente el origen etimológico de joven, del latín Iuvenis, que en el mundo romano se refería a la etapa entre los 30-45 años, es iuvare 'ayudar y sostener": ser el apoyo productivo de la sociedad, mientras que adulescens (el que adolece de algo -quien todavía está creciendo-) correspondía a los 18-29 años.

En la modernidad, al contrario, la juventud evoca más bien incompletitud. Los jóvenes son ciudadanos en transición a la vida adulta; en preparación para su incorporación al mercado de trabajo; ciudadanos que, al no estar plenamente integrados en el mercado de trabajo, no contribuyen ni participan enteramente de la propiedad social, para utilizar los atributos que Méda confiere al trabajo como institución central de las sociedades modernas.

No obstante, sabemos, muchos trabajadores que ya no son tan jóvenes, lo hacen precariamente. De hecho, el origen etimológico de precario viene de 'plegaria', que significa justamente "pedir ayuda". Trabajo precario parece ser así una contradicción en los términos. El trabajo precario, como forma excepcional de trabajo frente al trabajo normal, el trabajo estable, se concebía en los años ochenta y noventa como una ayuda a la inserción de los jóvenes en el mercado de trabajo, pero se ha terminado instalando como el carácter normal trabajo, acompañando a aquéllos jóvenes en sus trayectorias laborales al alcanzar la edad adulta.

De tal forma que ser joven no sólo ha perdido, con la modernidad, su antigua connotación de señalar la madurez de alguien autosuficiente capaz de ser un soporte para la sociedad, sino que la inseguridad y la dependencia características de las etapas de inserción al mundo del trabajo se han extendido a amplias clases de la población trabajadora, para las que, de alguna manera, el paso a la participación y la integración plenas y definitivas en la sociedad es una meta que siempre se desplaza [Castel, 2003].

El cambio hacia un modelo de vía baja de desarrollo ha supuesto una intensificación de los mecanismos de reproducción de clase (desciende la movilidad de clase ascendente e incrementa la descendente)-, que se acompañan de un incremento de las desigualdades sociales: mientras que en 2006 el $10 \%$ más rico disfrutaba de una renta 10 veces superior a la del 10\% más pobre, en 2015 esta diferencia fue de 15 veces (el 10\% más 
rico posee más del $40 \%$ de la riqueza ${ }^{16}$. Convirtiendo a España en el cuarto país más desigual de Europa). Pues el modelo productivo basado en la intensificación del trabajo y el abaratamiento de costes laborales requiere de una vinculación cada vez más directa entre carga de trabajo individual y salario percibido (mercantilización de las relaciones laborales), con lo que se intensifica la individualización de las relaciones sociales y da lugar a procesos de culpabilización personal y de psicologización de los conflictos de clase. A medida que se amplían y refuerzan los sistemas meritocráticos de asignación de empleos, tanto en el sistema educativo como en los servicios públicos de empleo, se incrementa el grado de responsabilidad personal en el estatus alcanzado.

Pero el recetario neoliberal aplicado para "salir de la crisis" sigue incidiendo en un modelo basado en la esquilmación de las nuevas generaciones. Los jóvenes son uno de los colectivos que más han sufrido la crisis en España, en la última EPA de 2016 la tasa de paro de los jóvenes entre 16 y 29 años todavía es del 31'5\%, la de temporalidad del $59^{\prime} 1 \%$ y la de tiempo parcial de $25^{\prime} 5 \%$. Un informe del Centro Reina Sofía sobre "Adolescencia y Juventud" afirma que "el 53\% de los jóvenes españoles entre 16 y 29 años cree que en los próximos dos años tendrá que irse de España por motivos laborales $^{17}$. El hiperrealismo con que los jóvenes perciben su futuro es corroborado por los datos de evolución de la población activa: en el año 2002 había 8.443 mil activos menores de 35 años, y suponían un 44\% de la población activa, en el año 2007 ascendieron a 9.286 mil (un 42\%), pero en el año 2016 sólo son 6.380 mil, un $28 \%$ de la población activa.

O sea, hoy hay 3 millones de activos jóvenes menores de 35 años menos que hace nueve años. Esta pérdida se ha debido a la caída de las tasas de actividad (especialmente entre los menores de 24 años que ha pasado entre 2007 y 2016 de $34 \%$ a $15 \%$ para los de 1620 años y de $70 \%$ a $57 \%$ para los de 20-24 años), pero fundamentalmente a la ya señalada caída de las tasas de fecundidad iniciada hace 30 años, y sobre todo, a la emigración económica hacia Centro-Europa y América Latina. El dato de la emigración económica es muy difícil de conocer debido a lo reciente del fenómeno y a la ausencia de investigaciones y registros actualizados, aunque según Eurostat al menos 617.201 jóvenes habrían salido a trabajar fuera de España desde el inicio de la crisis ${ }^{18}$. No obstante, si bien no se sabe con exactitud el número de jóvenes que han marchado fuera, sí sabemos los que faltan dentro: en 2012 la población total de jóvenes menores de 35 años sumaba 12.182 mil. En el año 2007, 12.393 mil. Y en el año 2016 se había reducido en esos mismos tres millones (9.491 mil).

De forma que la Juventud como institución de reproducción y legitimación de la desigualdad social no sólo ha tenido, sino que está teniendo actualmente un papel esencial en la citada transformación del modelo productivo. Las generaciones nacidas en las grandes reformas laborales de los años ochenta y noventa fueron la coartada ideológica necesaria para que, por medio de su incorporación al mundo del trabajo se operara la transformación del mercado de trabajo. Hoy, sin embargo, se oculta ese pasado sustituyéndolo por otro relato en el que precisamente se apunta a las prácticas y

16 Oxfam (2016): Bajan los salarios, crece la desigualdad. Informe de Oxfam Intermón, $\mathrm{n}^{\circ}$ 40, Noviembre de 2016. www.oxfamintermon.org [10-12-2016]

17 “EL 53\% DE LOS JÓVENES CREE QUE SE IRÁ DE ESPAÑA POR TRABAJO”, 20 Minutos, Madrid, 27-10-16 pg. 5

${ }^{18}$ http://ec.europa.eu/eurostat/statisticsexplained/index.php/Migration_and_migrant_population_statistics/es 
actitudes de cierta parte de la juventud como una las causas de la crisis acual, presentando los problemas que afectan hoy a los que han dejado de ser jóvenes, en cierto modo, como los efectos de sus decisiones erróneas en el pasado y de su falta de previsión.

Así es, se ha construido toda una etiología generalizante sobre los hándicaps caracteriológicos de las nuevas generaciones: desde la falta de activación y capacidad de emprendimiento en un contexto dominado por nuevas formas de trabajar y producir más creativas, flexibles e innovadoras, hasta la falta de adaptabilidad o empleabilidad ante los acelerados cambios de la oferta de puestos. Ya sea entre los trabajadores sin cualificación reglada, hoy en desempleo, que dejaron inconscientemente sus estudios atraídos por los cantos de sirena de sectores productivos que requerían trabajo manual y poco cualificado como los servicios y la construcción. Ya sea por trabajadores en desempleo -o con trabajos precarios-, bien sobrecualificados, o cuyas cualificaciones se han quedado obsoletas-, que no supieron adaptar sus estrategias formativas a las necesidades de la demanda, o que no son capaces de reciclarse hoy para ocupar nuevos nichos y yacimientos de empleo.

En nuestro proyecto de investigación actual Retos y alternativas a la precarización del trabajo y la vida en la crisis actual, 2005-2014 y que profundiza en el análisis de los efectos de la precarización del empleo y la vida social ("RETOSCRISIS", financiado por el Programa Nacional de I+D+I. Ref.: CSO2013-43666-R), estamos encontrando algunas manifestaciones de estos fenómenos de precarización y auto-culpabilización por parte de los propios jóvenes.

Concretamente, dos de los estudios de caso se centran en la situación de algunos jóvenes tras la crisis en dos contextos diferenciados aunque análogos en algunos aspectos clave. El Caso de la precariedad y el desempleo de los Jóvenes en Coslada y en Castilla La Mancha. Dos regiones que fueron protagonistas del crecimiento económico español del cambio de siglo al especializar sus economías en sectores productivos que, por una parte, contribuyeron con un peso específico al crecimiento del PIB, y por otra, fueron un atractivo de empleo fácil y bien remunerado para muchos jóvenes: los servicios y la logística en el caso de Coslada y la construcción y la agricultura -aunque en menor medida- en Castilla La Mancha.

Coslada es un gran municipio de la periferia metropolitana del sur de Madrid. Una región de antigua tradición industrial pero que fue radicalmente tercerizada en los años noventa, especializándose en la logística y el transporte, dada su localización estratégica como punto nodal de diferentes vías de transporte nacionales. Actividades que, por su carácter altamente dependiente de la actividad industrial y comercial han sido muy afectadas por la "gran recesión económica" ". Por su parte, en la comunidad de Castilla La Mancha sectores como la construcción y las actividades del sector primario y terciario de bajo valor añadido tuvieron un desarrollo muy alto en las décadas previas a la crisis. Ramas que hoy también han sido muy afectadas por la recesión económica.

\footnotetext{
${ }^{19}$ El parque de vehículos pesados autorizados para el transporte de mercancías se redujo de los 400.000 a los 349.593 sólo entre 2007 y 2012. El número de operaciones de transporte pasó en esos años de los 332 millones a los 200. Lo que ha supuesto pasar de 2.408.978 toneladas transportadas a las 1.466 .502 en 2011. Ya en el año 2008 para la ocupación conductor de camión hubo 49.418 demandas de empleo para sólo 3.000 ofertas, y en el año 2011, el número de demandas alcanzó la cifra de 70.625, mientras el número de ofertas apenas llegó a las 1.737. De tal manera que en el sector del transporte terrestre y las actividades anexas, se cerraron unas 14.500 empresas durante el bienio 2008-2010, y el valor añadido bruto de la rama de actividad Transporte y Comunicaciones, que venía suponiendo un $7 \%$ anual del total nacional hasta el año 2007, bajó un punto sólo en tres años.
} 
Muchos de estos jóvenes que dejaron la escuela atraídos por el trabajo fácil y los salarios relativamente atractivos que ofrecía el transporte o la construcción, hoy se lamentan de haber abandonado prematuramente la escuela ("yo era un cabeza loca") o por dejarse llevar por lo que hacían sus pares ("esto me ha pasao por juntarme con quien no debia"), haciéndose responsables, en última instancia, de haberlo "echado todo a perder" en sus años de formación. Otros achacan su situación actual de desempleo y falta de oportunidades a ciertas taras personales vinculadas a su escasa cualificación como no "valer para estudiar", y sólo valer "para trabajar". Y otros, por supuesto, a toda una serie de decisiones erróneas en el campo del ocio y el consumo, como el haber contraído hipotecas y préstamos para adquirir viviendas o automóviles que estaban por encima de sus posibilidades de gasto real, y que hoy han tenido que malvender o devolver. Jóvenes con 30-35 años hoy que abandonaron la educación secundaria atraídos por ofertas de trabajo que no exigían una gran cualificación pero muy bien remuneradas y que hoy están sin empleo y sin cualificación ${ }^{20}$. Pero también hemos dado con el perfil de mujeres jóvenes que con más frecuencia "hicieron todo bien" (es decir aprobaron la ESO, cursaron bachillerato y muchas sacaron títulos universitarios), y que se encuentran hoy en la necesidad de "ocultar" en sus currículums la formación alcanzada, como estrategia para acceder a un empleo: "el que sea" [Candela, 2016].

En muchos casos, a los jóvenes de zonas rurales o de zonas urbanas deprimidas, de familias de rentas modestas (o en lo últimos años, excluidas socialmente) con poco capital social y cultural, el sistema educativo no les está proporcionándoles el soporte y las oportunidades necesarias para un cambio de alternativas, antes bien contribuye a reforzar y reproducir limitaciones de origen. Pues en buena medida las trayectorias exploradas refuerzan la tesis de que el abandono escolar en la enseñanza secundaria responde, en parte, a la naturaleza "selectiva" de esta etapa educativa en confrontación con la tendencia más inclusiva de la escuela primaria. En el segundo ciclo de la ESO y en el Bachillerato se etiqueta, clasifica y separa al alumnado que teóricamente vale para seguir estudiando y al que no, se le condena socialmente al "abandono". Y aun los que superan la dinámica selectiva del bachillerato, deben pasar la "selectividad" (o PAEG) con mayúsculas, la barrera de acceso a la Universidad que condiciona hasta las vocaciones, en función de los resultados de las calificaciones obtenidas.

A los que sí logran encontrar un empleo les esperan altos niveles de temporalidad y precariedad, que afectan precisamente a las ocupaciones de más bajo nivel de cualificación (peones y camareros), concentrando tasas del $46 \%$ y $18 \%$ respecto al total de ocupaciones con mayor contratación temporal ${ }^{21}$.

En última instancia, la crisis económica mundial, y la especial virulencia con la que ha afectado a países de la semiperiferia sur europea como España, se ha mostrado, antes que nada, como un revelador de la insostenibilidad del modelo de desarrollo anterior y ha sido el catalizador de la transición al nuevo modelo de acumulación basado en los bajos costes y la intensificación del trabajo.

\footnotetext{
${ }^{20}$ Por ejemplo, en el caso de Castilla La Mancha Si atendemos al nivel de estudios de los parados registrados, comprobamos que el 61,3\% pertenecen al subtotal "Primera Etapa de Educación Secundaria". En la contratación de menores de 30 años se destaca la superior masculina, con un peso del 61,65\%. El tramo de edad más activo en las contrataciones es el 25 a 29 años.
}

${ }^{21}$ Observatorio de las Ocupaciones de Castilla La Mancha, 2015: 52. 


\section{Referencias:}

Bilbao, Andrés (1989): “La utilización ideológica de los jóvenes”, en Torregrosa et al., 1989, pp.59-65.

-Bourdieu, Pierre y Passeron, Jean-Claude (1970): La reproduction. Elèments pour une théorie du système d'enseignement, Editions de Minuit, Paris

-CASTEL, R. (2003): L'insecurité sociale. Qu'est-ce qu'etre protegé. París, Eds. du Seuil.

-Castillo, Juan José (1986) "Política ocupacional: débiles y fuertes. Tribuna: El problema del paro", El País, 8 de abril de 1986.

-Castillo, Juan José (1987): "Crisis del trabajo y cambios sociales", número monográfico de la Revista Española de Investigaciones Sociológicas, REIS, 38, pp. 720, "Introducción".

-Castillo, Juan José (1989): "Transformaciones productivas, crisis del trabajo y comportamientos sociales: los jóvenes por ejemplo", en Torregrosa et al. 1989, pp. 6983.

-Castillo, J. y López Calle, P. (2004): Los hijos de las Reformas Laborales. Vivienda, formación y empleo de los jóvenes en la Comunidad de Madrid, UGT, Madrid

-Castillo, J. y López Calle, P. (2007) “Una Generación Esquilmada: los efectos de las reformas laborales en el trabajo y la vida de los jóvenes". Sociedad y Utopía, Revista de Ciencias Sociales. $\mathrm{n}^{\circ} 29$, pp 273-313

-Candela, P. (2016): "Sobreviviendo a la crisis: trayectorias formativas y laborales de jóvenes castellano-manchegos que soñaron con el ladrillo", en Revista del Ministerio de Empleo y Seguridad Social, núm. 120, pp. 93-118.

-Instituto de la Juventud (2001): Juventud española, 2000, Estudio cuatrianual de la juventud española, INJUVE, $33 \mathrm{pp}$.

-López Calle, P (2012): "Alicia y Yo. Una perspectiva socio-clínica sobre explotación y alienación del trabajo en los nuevos modelos productivos", Intersubjetivo. Revista de psicoterapia, psicoanalítica y salud. Vol. 12, pp. 166-188.

-López Calle, P. e Ibáñez Rojo, R. (2012): "Financiarización y relación salarial: estrategias de rentabilidad y conflictos de clase en la semiperiferia del sistema-mundo", en Alonso, L.E y Fernández Rodríguez, C. (eds.): La financiarización de las relaciones salariales. Una perspectiva internacional. La Catarata, Madrid, 2012, Cap. VIII, pp. $186-210$

-Martín Criado, E. (1998): Producir la juventud. Crítica de la sociología de la juventud, Tres Cantos (Madrid), Ediciones Itsmo.

-Martín Criado, E. (2010): La escuela sin funciones. Crítica de la sociología de la educación crítica, Barcelona, Ediciones Bellatera. 
-Torregrosa, José Ramón; Berguere Dezaphi, Joelle y Alavaro Estramiana, José Luis (Eds.) (1989): Juventud, trabajo y desempleo: un análisis psicosociológico. Ministerio de Trabajo y Seguridad Social, Madrid, 567pp.

-Willis, Paul (1988): Aprendiendo a trabajar. Cómo los chicos de la clase obrera consiguen trabajos de clase obrera, Akal, Madrid.

-Zárraga, José Luis de (1985): Informe juventud en España. La inserción de los jóvenes en la sociedad. Ministerio de Cultura. Instituto de la Juventud, 403 pp. 